\title{
Analysis of three-dimensional boundary-layer nanofluid flow and heat transfer over a stretching surface by means of the homotopy analysis method
}

\author{
Qingkai Zhao ${ }^{1 *}$, Hang $X u^{1}$ and Tao Fan ${ }^{2}$
}

\author{
"Correspondence: \\ qkzhao@sjtu.edu.cn \\ 'State Key Lab of Ocean \\ Engineering, School of Naval \\ Architecture, Ocean and Civil \\ Engineering, Shanghai Jiao Tong \\ University, Shanghai, 200240, \\ P.R. China \\ Full list of author information is \\ available at the end of the article
}

\begin{abstract}
In this paper, an investigation is made of the three-dimensional flow and heat transfer of a nanofluid in the boundary-layer region over a flat sheet stretched continuously in two lateral directions. With the help of a series of similarity transformations, this problem is reduced to a set of three coupled differential equations. The homotopy analysis method (HAM) is then applied to derive the explicit solutions for both the velocity and the temperature distributions. A mathematical analysis shows that these solutions decay exponentially at far field. Besides, the influences of the nanoparticle volume fraction $\phi$ on the velocity and temperature profiles, as well as the reduced local skin friction coefficients and the reduced local Nusselt numbers are studied. It is found that the heat transfer conductivity of the nanofluid is superior to that of the pure fluids.
\end{abstract}

Keywords: boundary layer; nanofluid flow; stretching; heat transfer; explicit solution

\section{Introduction}

Flow and heat transfer in the boundary-layer region near a stretching flat surface have been intensively investigated by many researchers during the past several decades owing to their wide applications in a number of industrial processes, for example, polymer extrusion, wire drawing, drawing of plastic films and artificial fibers, hot rolling, glassfiber, metal extrusion, metal spinning, and so on. Crane [1] investigated the laminar flow over a stretching flat sheet in an otherwise ambient fluid. From then on, many researchers have made contributions to various aspects of this problem such as the effects of the arbitrary velocity of the stretching sheet, magnetic field, blowing or suction, variable thermal conductivity or wall temperature. Typical works can be found in Gupta and Gupta [2], Chakrabarti and Gupta [3], Carragher and Crane [4], Dutta et al. [5], Jeng et al. [6], Dutta [7], Andersson [8], Chiam [9], Vajravelu and Hadjinicolaou [10], etc. It should be noted that Wang [11] initiated the investigation of the three-dimensional boundary-layer flow caused by a stretching flat sheet in two lateral directions in an otherwise ambient fluid. Hayat et al. [12] considered the three-dimensional boundary-layer flow over a stretching surface immersed in a viscoelastic fluid. Takhar et al. [13] investigated the unsteady three-dimensional MHD boundary-layer flow and heat transfer caused by an impulsive

(c) 2015 Zhao et al.; licensee Springer. This article is distributed under the terms of the Creative Commons Attribution 4.0 International License (http://creativecommons.org/licenses/by/4.0/), which permits unrestricted use, distribution, and reproduction in any medium, provided you give appropriate credit to the original author(s) and the source, provide a link to the Creative Commons license, and indicate if changes were made. 
motion of a stretching surface in two lateral directions and a suddenly increasing surface temperature from that of the surrounding fluid.

Nanofluids are expected to be a new generation of heat transfer fluids, since they have higher thermal conductivity and single-phase heat transfer coefficients as compared with those of traditional heat transfer fluids. Several types of mathematical models have been proposed for the prediction of the behaviors of nanofluids. One type is the homogeneous models, which were initially proposed by Choi [14] and then developed by Maïga et al. [15]. By means of this approach, it is very convenient to extend the conventional conservation equations for pure fluids to nanofluids. Therefore, all traditional heat transfer correlations involving the computation of the thermophysical properties could be suitable for nanofluids as well. The deficiency of these models is that the obtained results are incompatible with the experimental observation as a large value of the solid volume fraction $\phi$ is chosen. The valid region for $\phi$ is usually limited to $0 \leq \phi \leq 0.3$. The dispersion models are another usual approach used for modeling the nanofluids, which were first suggested by Xuan and Roetzel [16]. In this approach, the higher thermal conductivity and the dispersion of nanoparticles are assumed to be the two dominant factors for convective heat transfer enhancement. They therefore treated the effect of the relative velocity between the base fluid and the nanoparticles as a perturbation of the energy equation and then introduced an empirical dispersion coefficient to describe the heat transfer enhancement. Unfortunately, in this approach, the heat transfer enhancement due to nanoparticle dispersion is too small to be considered in nanofluids. The third approach used for modeling nanofluids was proposed by Buongiorno [17] based on the mechanics of the nanoparticle/base-fluid relative velocity. In this model, he, respectively, analyzed the seven slip mechanisms for nanofluids including inertia, Brownian diffusion, thermophoresis, diffusiophoresis, Magnus effect, fluid drainage, and gravity settling. He then drew the conclusion that the Brownian diffusion and the thermophoresis are the two most important factors, provided the turbulent effects are not considered. Using the Buongiorno [17] mathematical model, Nield and Kuznetsov [18, 19] extended the well-known Cheng and Minkowycz problem [20] to nanofluids with various physical conditions. Their theoretical results verified the validity of the Buongiorno [17] model for dilute nanofluids. One blemish of this approach is that the physical parameters such as the coefficients of the Brownian diffusion and the thermophoresis are extremely hard to measure experimentally. Another blemish is that, with this model, many well-constructed nonlinear problems in Newtonian fluids cannot be formulated for nanofluids even if the same initial and boundary conditions are given with the boundary-layer approximations and the similarity transformations. This provides the theoretical researchers with difficulties on resolving them numerically or analytically. Recently, some efforts have been made toward understanding of flow and heat transfer characteristics of nanofluids. Among those investigations, some researchers applied the homogeneous models [21-25] to analyze and simplify their physical problems, while other researchers utilized the Buongiorno model [26-29] in their theoretical analysis and numerical simulations.

The present paper considers the steady laminar flow and heat transfer of a nanofluid in the boundary-layer region near a flat surface stretched continuously in two lateral directions. A homogeneous nanofluid model is employed here for simplification of the physical problem. With this approach, a set of three coupled differential equations with their appropriate boundary conditions are obtained by means of some similarity transformations. 
The homotopy analysis method (HAM) [30] is introduced to give explicit solutions with high accuracy for those differential equations. An asymptotic analysis is then provided to examine the mathematical behavior of the reduced velocity and temperature profiles at far field. As far as we know, this problem has not been considered before so that the results are new and original. Note that nanofluids have superior heat transfer properties as compared to conventional heat transfer fluids. This makes nanofluids very attractive for a number of potential engineering applications involved in flow and heat transfer over flat sheets, such as in the design of cooling and heat exchange for nuclear systems, in the configuration of collector of solar energy, and in the thermal processing regarding to the production of paper, polymeric sheets, insulating materials, fine-fiber mattes, etc. Nanofluids provide us with a new opportunity for making new high-efficiency systems and improving the existing systems in industrial applications.

\section{Mathematical description}

We consider the steady, three-dimensional boundary-layer flow and heat transfer of a nanofluid in the vicinity of a stretching flat surface in an otherwise quiescent fluid. It is assumed that the nanofluid is incompressible and the flow is laminar. The physical sketch and the coordinate system is plotted in Figure 1. As shown by this figure, the flat surface is stretching continuously in both $x$ - and $y$-directions with the velocities $u_{w}=a x$ and $v_{w}=b y$, respectively. The surface temperature is $T_{w}$ and the ambient temperature is $T_{\infty}$, where $T_{w}$ and $T_{\infty}$ are two constants, with $T_{w}>T_{\infty}$. The nanofluid is the suspension of solid particles in nano-scale in a base fluid. The nanoparticles considered here are $\mathrm{Cu}$, and the base fluid is water. The thermophysical properties of the base fluid and the nanoparticles are given in Table 1. Using the mathematical model for nanofluids proposed by Choi [14], the governing equations are given by (refer to [31])

$$
\begin{aligned}
& \frac{\partial u}{\partial x}+\frac{\partial v}{\partial y}+\frac{\partial w}{\partial z}=0 \\
& u \frac{\partial u}{\partial x}+v \frac{\partial u}{\partial y}+w \frac{\partial u}{\partial z}=\frac{\mu_{\mathrm{nf}}}{\rho_{\mathrm{nf}}} \frac{\partial^{2} u}{\partial z^{2}}
\end{aligned}
$$

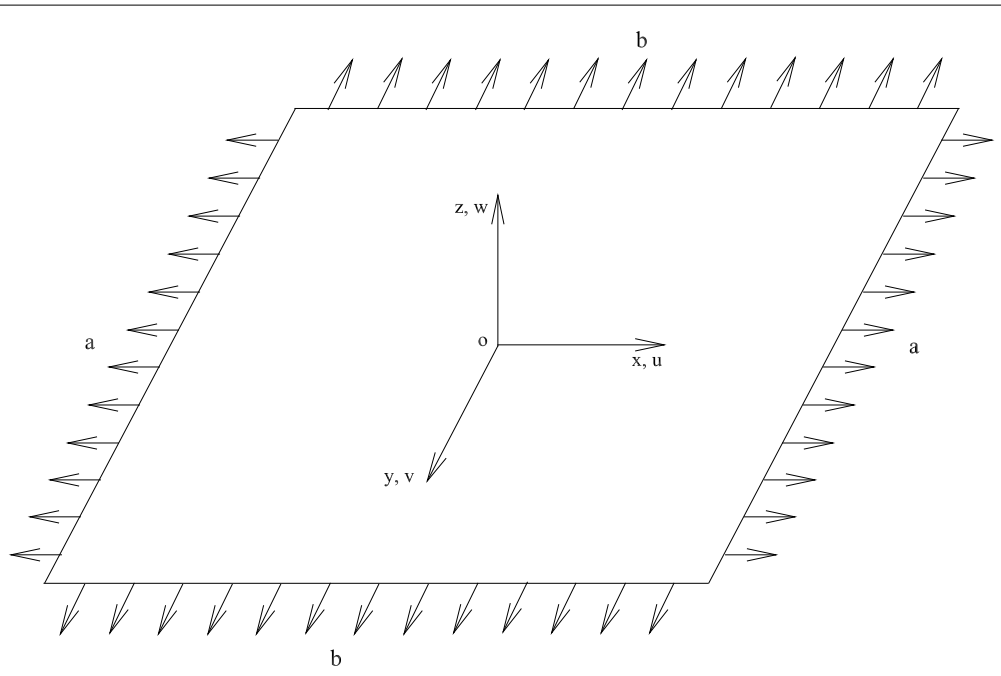

Figure 1 A physical sketch and the coordinate system. 
Table 1 Thermophysical properties of base fluid and nanoparticles [33]

\begin{tabular}{lcrrc}
\hline Physical properties & Base fluid & \multicolumn{1}{c}{$\mathbf{C u}$} & $\mathbf{A l}_{\mathbf{2}} \mathbf{O}_{\mathbf{3}}$ & $\mathbf{T i O}_{\mathbf{2}}$ \\
\hline$C_{p}(\mathrm{~J} / \mathrm{kg} \mathrm{K})$ & 4,179 & 385 & 765 & 686.2 \\
$\rho\left(\mathrm{kg} / \mathrm{m}^{3}\right)$ & 997.1 & 8,933 & 3,970 & 4,250 \\
$k(\mathrm{~W} / \mathrm{mK})$ & 0.613 & 400 & 40 & 8.9538 \\
\hline
\end{tabular}

$$
\begin{aligned}
& u \frac{\partial v}{\partial x}+v \frac{\partial v}{\partial y}+w \frac{\partial v}{\partial z}=\frac{\mu_{\mathrm{nf}}}{\rho_{\mathrm{nf}}} \frac{\partial^{2} v}{\partial z^{2}}, \\
& u \frac{\partial T}{\partial x}+v \frac{\partial T}{\partial y}+w \frac{\partial T}{\partial z}=\alpha_{\mathrm{nf}} \frac{\partial^{2} T}{\partial z^{2}},
\end{aligned}
$$

subject to the boundary conditions

$$
\begin{aligned}
& u(x, y, 0)=u_{w}=a x, \quad v(x, y, 0)=v_{w}=b y, \quad w(x, y, 0)=0, \\
& T(x, y, 0)=T_{w}, \quad u(x, y, \infty)=v(x, y, \infty)=0, \quad T(x, y, \infty)=T_{\infty} .
\end{aligned}
$$

Here $(u, v, w)$ are the velocity components along the axes $(x, y, z), T$ is the temperature of the nanofluid, $a$ and $b$ are positive constants. $\mu_{\mathrm{nf}}, \rho_{\mathrm{nf}}$, and $\alpha_{\mathrm{nf}}$ are the effective viscosity of the nanofluid, the effective density of the nanofluid, and the effective thermal diffusivity of the nanofluid, respectively, which are defined as

$$
\begin{aligned}
& \mu_{\mathrm{nf}}=\frac{\mu_{\mathrm{f}}}{(1-\phi)^{2.5}}, \quad \rho_{\mathrm{nf}}=(1-\phi) \rho_{\mathrm{f}}+\phi \rho_{\mathrm{s}}, \quad \alpha_{\mathrm{nf}}=\frac{k_{\mathrm{nf}}}{\left(\rho C_{p}\right)_{\mathrm{nf}}} \\
& \left(\rho C_{p}\right)_{\mathrm{nf}}=(1-\phi)\left(\rho C_{p}\right)_{\mathrm{f}}+\phi\left(\rho C_{p}\right)_{\mathrm{s}}, \quad \frac{k_{\mathrm{nf}}}{k_{\mathrm{f}}}=\frac{\left(k_{\mathrm{s}}+2 k_{\mathrm{f}}\right)-2 \phi\left(k_{\mathrm{f}}-k_{\mathrm{s}}\right)}{\left(k_{\mathrm{s}}+2 k_{\mathrm{f}}\right)+\phi\left(k_{\mathrm{f}}-k_{\mathrm{s}}\right)}
\end{aligned}
$$

where $\phi$ is the solid volume fraction of the nanofluid, $\mu$ is the viscosity, $\rho$ is the density, $k$ is the thermal conductivity, $\rho C_{p}$ is the heat capacitance, the subscript 'nf' represents the nanofluid, 'f' represents the base fluid, and 's' represents the nanoparticles, respectively. It should be pointed out that the expression for $\mu_{\mathrm{nf}}$ in (7) is given by Brinkman [32] and the effective thermal conductivity of the nanofluid $k_{\mathrm{nf}}$ is approximated by the MaxwellGarnetts model [15], which is found to be appropriate for studying heat transfer enhancement using nanofluids, and the shape of the nanoparticles is restricted to spherical ones.

We introduce now the following similarity transformations:

$$
\begin{aligned}
& \eta=z\left(\frac{a}{v_{\mathrm{f}}}\right)^{1 / 2}, \quad u(x, y, z)=a x f^{\prime}(\eta), \quad v(x, y, z)=a y g^{\prime}(\eta), \\
& w(x, y, z)=-\left(a v_{\mathrm{f}}\right)^{1 / 2}(f+g), \quad T(x, y, z)=T_{\infty}+\left(T_{w}-T_{\infty}\right) \theta(\eta),
\end{aligned}
$$

where primes denote differentiation with respect to $\eta$ and $v_{\mathrm{f}}$ is the kinematic viscosity of the base fluid. Substituting the similarity variables (8) into (1)-(4), it is found that the continuity equation (1) is automatically satisfied, and (2)-(4) are reduced to

$$
\begin{aligned}
& \varepsilon_{1} f^{\prime \prime \prime}+(f+g) f^{\prime \prime}-\left(f^{\prime}\right)^{2}=0, \\
& \varepsilon_{1} g^{\prime \prime \prime}+(f+g) g^{\prime \prime}-\left(g^{\prime}\right)^{2}=0, \\
& \frac{\varepsilon_{2}}{P r} \theta^{\prime \prime}+(f+g) \theta^{\prime}=0,
\end{aligned}
$$


subject to the following boundary conditions:

$$
\begin{aligned}
& f(0)=g(0)=0, \quad f^{\prime}(0)=1, \quad g^{\prime}(0)=c, \quad \theta(0)=1, \\
& f^{\prime}(\infty)=0, \quad g^{\prime}(\infty)=0, \quad \theta(\infty)=0,
\end{aligned}
$$

where $P r=v_{\mathrm{f}} / \alpha_{\mathrm{f}}$ is the Prandtl number, $c=b / a$ is the ratio of the surface velocity gradients along the $y$-and $x$-directions; $\varepsilon_{1}$ and $\varepsilon_{2}$ are two constants relating to the properties of the nanofluid, defined by

$$
\begin{aligned}
& \varepsilon_{1}=\frac{1}{(1-\phi)^{2.5}\left[(1-\phi)+\phi \rho_{\mathrm{s}} / \rho_{\mathrm{f}}\right]}, \\
& \varepsilon_{2}=\frac{k_{\mathrm{nf}} / k_{\mathrm{f}}}{(1-\phi)+\phi\left(\rho C_{p}\right)_{\mathrm{s}} /\left(\rho C_{p}\right)_{\mathrm{f}}} .
\end{aligned}
$$

The physical quantities of practical interest are the local skin friction coefficients $C_{f x}$ and $C_{f y}$ and the local Nusselt numbers $N u_{x}$ and $N u_{y}$, which are defined by

$$
\begin{aligned}
& C_{f x}=\frac{\tau_{x}}{\rho_{\mathrm{f}} u_{w}^{2}}, \quad C_{f y}=\frac{\tau_{y}}{\rho_{\mathrm{f}} v_{w}^{2}}, \\
& N u_{x}=\frac{x q_{w}}{k_{\mathrm{f}}\left(T_{w}-T_{\infty}\right)}, \quad N u_{y}=\frac{y q_{w}}{k_{\mathrm{f}}\left(T_{w}-T_{\infty}\right)},
\end{aligned}
$$

where $\tau_{x}$ and $\tau_{y}$ are the shear stresses in the $x$-and $y$-directions, respectively, $q_{w}$ is the heat flux from the stretching sheet. For the current case, the three quantities can be written as

$$
\tau_{x}=-\mu_{\mathrm{nf}}\left(\frac{\partial u}{\partial z}\right)_{z=0}, \quad \tau_{y}=-\mu_{\mathrm{nf}}\left(\frac{\partial v}{\partial z}\right)_{z=0}, \quad q_{w}=-k_{\mathrm{nf}}\left(\frac{\partial T}{\partial z}\right)_{z=0} .
$$

Substituting (8) into (14) and using (15), we obtain

$$
\begin{aligned}
& \operatorname{Re}_{x}^{1 / 2} C_{f x}=-\frac{f^{\prime \prime}(0)}{(1-\phi)^{2.5}}, \quad \operatorname{Re}_{y}^{1 / 2} C_{f y}=-\frac{g^{\prime \prime}(0)}{(1-\phi)^{2.5} c^{3 / 2}}, \\
& N u_{x}=-\operatorname{Re}_{x}^{1 / 2} \frac{k_{\mathrm{nf}}}{k_{\mathrm{f}}} \theta^{\prime}(0), \quad N u_{y}=-\operatorname{Re}_{y}^{1 / 2} \frac{k_{\mathrm{nf}}}{k_{\mathrm{f}} \mathcal{C}^{1 / 2}} \theta^{\prime}(0),
\end{aligned}
$$

where $R e_{x}=\left(u_{w} x\right) / \nu_{\mathrm{f}}$ and $R e_{y}=\left(v_{w} y\right) / \nu_{\mathrm{f}}$ are the local Reynolds numbers in the $x$ - and $y$-direction, respectively.

\section{Explicit solutions}

The explicit solutions for $f(\eta), g(\eta)$, and $\theta(\eta)$ can be formulated with the help of the homotopy analysis method (HAM) [30], as illustrated hereafter. In the framework of the HAM, we are able to express $f(\eta), g(\eta)$, and $\theta(\eta)$ by an infinite number of sub-functions in the following form:

$$
f(\eta)=\sum_{k=0}^{\infty} f_{k}(\eta), \quad g(\eta)=\sum_{k=0}^{\infty} g_{k}(\eta), \quad \theta(\eta)=\sum_{k=0}^{\infty} \theta_{k}(\eta)
$$


where

$$
\begin{aligned}
& f_{k}(\eta)=\hat{A}_{0,0}^{k}+\sum_{j=1}^{k+1} \sum_{i=0}^{k+1} \sigma_{i, j}^{k} \hat{A}_{i, j}^{k} \eta^{i} \exp (-j \lambda \eta), \\
& g_{k}(\eta)=\hat{B}_{0,0}^{k}+\sum_{j=1}^{k+1} \sum_{i=0}^{k+1} \sigma_{i, j}^{k} \hat{B}_{i, j}^{k} \eta^{i} \exp (-j \lambda \eta), \\
& \theta_{k}(\eta)=\sum_{j=1}^{k+1} \sum_{i=0}^{k+1} \sigma_{i, j}^{k} \hat{C}_{i, j}^{k} \eta^{i} \exp (-j \lambda \eta) .
\end{aligned}
$$

According to the rule of the solution expressions and boundary conditions (12) and (13), we choose the initial approximations as follows:

$$
\begin{aligned}
& f_{0}(\eta)=\frac{1}{\lambda}\left(1-e^{-\lambda \eta}\right), \\
& g_{0}(\eta)=\frac{c}{\lambda}\left(1-e^{-\lambda \eta}\right), \\
& \theta_{0}(\eta)=e^{-\lambda \eta} .
\end{aligned}
$$

Choosing the auxiliary linear operator as follows:

$$
\begin{aligned}
& \mathscr{L}_{f}=\frac{\partial^{3}}{\partial \eta^{3}}-\lambda^{2} \frac{\partial}{\partial \eta}, \\
& \mathscr{L}_{g}=\frac{\partial^{3}}{\partial \eta^{3}}-\lambda^{2} \frac{\partial}{\partial \eta}, \\
& \mathscr{L}_{\theta}=\frac{\partial^{2}}{\partial \eta^{2}}-\lambda^{2} \theta,
\end{aligned}
$$

where $\lambda$ is the spatial parameter, which is chosen based on the solution properties at infinity and can be used to controlled solution convergence. We have set $\lambda=1$ in our paper.

The corresponding coefficients in (18)-(20) are obtained by means of the HAM as

$$
\begin{aligned}
\hat{A}_{0,0}^{k}= & \chi_{k} \hat{A}_{0,0}^{k-1}+\sum_{j=2}^{k+1} \sum_{q=1}^{k+1} \Gamma_{q, j}^{k}\left[(j-1) \omega_{j, 0}^{q}-\frac{1}{\lambda} \omega_{j, 1}^{q}\right] \\
& -\sum_{q=0}^{k} \frac{1}{\lambda} \Delta_{q, 1}^{k} \omega_{1,1}^{q}+\sum_{j=2}^{k+1}(j-1) \Gamma_{0, j}^{k} \omega_{j, 0}^{0}, \\
\hat{A}_{0,1}^{k}= & \chi_{k} \sigma_{0,1}^{k-1} \hat{A}_{0,1}^{k-1}+\sum_{j=2}^{k+1} \sum_{q=1}^{k+1} \frac{1}{\lambda} \Gamma_{q, j}^{k} \omega_{j, 1}^{q}+\sum_{q=0}^{k} \frac{1}{\lambda} \Delta_{q, 1}^{k} \omega_{1,1}^{q}-\sum_{j=2}^{k+1} \sum_{q=0}^{k+1} j \Gamma_{q, j}^{k} \omega_{j, 0}^{q}, \\
\hat{A}_{0, j}^{k}= & 0 \quad(2 \leq j \leq k+1), \\
\hat{A}_{i, 1}^{k}= & \chi_{k} \sigma_{i, 1}^{k-1} \hat{A}_{i, 1}^{k-1}+\sum_{q=\max [0, i-1]}^{k} \Delta_{q, 1}^{k} \omega_{1, i}^{q} \quad(1 \leq i \leq k+1), \\
\hat{A}_{i, j}^{k}= & \chi_{k} \sigma_{i, j}^{k-1} \hat{A}_{i, j}^{k-1}+\sum_{q=i}^{k+1} \Gamma_{q, j}^{k} \omega_{j, i}^{q} \quad(1 \leq i \leq k+1,2 \leq j \leq k+1),
\end{aligned}
$$




$$
\begin{aligned}
& \hat{B}_{0,0}^{k}=\chi_{k} \hat{B}_{0,0}^{k-1}+\sum_{j=2}^{k+1} \sum_{q=1}^{k+1} \Theta_{q, j}^{k}\left[(j-1) \omega_{j, 0}^{q}-\frac{1}{\lambda} \omega_{j, 1}^{q}\right] \\
& -\sum_{q=0}^{k} \frac{1}{\lambda} \Lambda_{q, 1}^{k} \omega_{1,1}^{q}+\sum_{j=2}^{k+1}(j-1) \Theta_{0, j}^{k} \omega_{j, 0}^{0}, \\
& \hat{B}_{0,1}^{k}=\chi_{k} \sigma_{0,1}^{k-1} \hat{B}_{0,1}^{k-1}+\sum_{j=2}^{k+1} \sum_{q=1}^{k+1} \frac{1}{\lambda} \Theta_{q, j}^{k} \omega_{j, 1}^{q} \\
& +\sum_{q=0}^{k} \frac{1}{\lambda} \Lambda_{q, 1}^{k} \omega_{1,1}^{q}-\sum_{j=2}^{k+1} \sum_{q=0}^{k+1} j \Theta_{q, j}^{k} \omega_{j, 0}^{q} \\
& \hat{B}_{0, j}^{k}=0 \quad(2 \leq j \leq k+1), \\
& \hat{B}_{i, 1}^{k}=\chi_{k} \sigma_{i, 1}^{k-1} \hat{B}_{i, 1}^{k-1}+\sum_{q=\max [0, i-1]}^{k} \Lambda_{q, 1}^{k} \omega_{1, i}^{q} \quad(1 \leq i \leq k+1), \\
& \hat{B}_{i, j}^{k}=\chi_{k} \sigma_{i, j}^{k-1} \hat{B}_{i, j}^{k-1}+\sum_{q=i}^{k+1} \Theta_{q, j}^{k} \omega_{j, i}^{q} \quad(1 \leq i \leq k+1,2 \leq j \leq k+1), \\
& \hat{C}_{0,1}^{k}=\chi_{k} \sigma_{0,1}^{k-1} \hat{C}_{0,1}^{k-1}-\sum_{j=2}^{k+1} \sum_{q=0}^{k+1} \Psi_{q, j}^{k} \delta_{j, 0}^{q}, \\
& \hat{C}_{i, 1}^{k}=\chi_{k} \delta_{i, 1}^{k-1} \hat{C}_{i, 1}^{k-1}+\sum_{q=\max [0, i-1]}^{k} \Omega_{q, 1}^{k} \delta_{1, i}^{q} \quad(1 \leq i \leq k+1), \\
& \hat{C}_{0, j}^{k}=0 \quad(2 \leq j \leq k+1), \\
& \hat{C}_{i, j}^{k}=\chi_{k} \delta_{i, j}^{k-1} \hat{C}_{i, j}^{k-1}+\sum_{q=i}^{k+1} \Psi_{q, j}^{k} \delta_{j, i}^{q} \quad(1 \leq i \leq k+1,2 \leq j \leq k+1),
\end{aligned}
$$

with

$$
\begin{aligned}
& \chi_{k}= \begin{cases}0, & k=1, \\
1, & k>1,\end{cases} \\
& \sigma_{i, j}^{k}= \begin{cases}1, & 0 \leq i \leq k+1 \text { and } 1 \leq j \leq k+1, \\
0, & \text { other cases, }\end{cases} \\
& \omega_{n, j}^{q}= \begin{cases}\sum_{i=j}^{q+1} \frac{\delta_{n, j}^{q}}{\lambda^{i-j+1}}, & 0 \leq j \leq q+1 \text { and } n=1, \\
\sum_{i=j}^{q} \frac{\delta_{n, j}^{q}}{(n \lambda)^{i-j+1}}, & 0 \leq j \leq q \text { and } n>1, \\
0, & j=q+1 \text { and } n>1\end{cases}
\end{aligned}
$$

and

$$
\delta_{n, j}^{q}= \begin{cases}\frac{q !}{j !} \frac{1}{(2 \lambda)^{q-j+2}}, & 0 \leq j \leq q \text { and } n=1, \\ \frac{1}{2 \lambda(q+1)}, & j=q+1 \text { and } n=1, \\ \frac{1}{2 \lambda} \frac{q !}{j !}\left\{\frac{1}{[(n+1) \lambda]^{q-j+1}}-\frac{1}{[(n-1) \lambda]^{q-j+1}}\right\}, & 0 \leq j \leq q \text { and } n>1, \\ 0, & j=q+1 \text { and } n>1 .\end{cases}
$$


Other functions in the above recurrence formulas are given by

$$
\begin{aligned}
& \Gamma_{i, j}^{k}=\hbar_{f}\left(\varepsilon_{1} \hat{J}_{i, j}^{k-1}+\tilde{\mathcal{A}}_{i, j}^{k}+\tilde{\mathcal{B}}_{i, j}^{k}+\tilde{\mathcal{E}}_{i, j}^{k}+\tilde{\mathcal{F}}_{i, j}^{k}+\tilde{\mathcal{G}}_{i, j}^{k}\right), \\
& \Theta_{i, j}^{k}=\hbar_{g}\left(\varepsilon_{1} \hat{K}_{i, j}^{k-1}+\tilde{\mathcal{C}}_{i, j}^{k}+\tilde{\mathcal{D}}_{i, j}^{k}+\tilde{\mathcal{H}}_{i, j}^{k}+\tilde{\mathcal{I}}_{i, j}^{k}+\tilde{\mathcal{J}}_{i, j}^{k}\right), \\
& \Psi_{i, j}^{k}=\hbar_{\theta}\left(\frac{\varepsilon_{2}}{\operatorname{Pr}} \hat{I}_{i, j}^{k-1}+\tilde{\mathcal{K}}_{i, j}^{k}+\tilde{\mathcal{L}}_{i, j}^{k}+\tilde{\mathcal{M}}_{i, j}^{k}+\tilde{\mathcal{L}}_{i, j}^{k}\right), \\
& \Delta_{i, 1}^{k}=\hbar_{f}\left(\varepsilon_{1} \hat{J}_{i, j}^{k-1}+\tilde{\mathcal{B}}_{i, 1}^{k}+\tilde{\mathcal{G}}_{i, 1}^{k}\right), \\
& \Lambda_{i, 1}^{k}=\hbar_{g}\left(\varepsilon_{1} \hat{K}_{i, 1}^{k-1}+\tilde{\mathcal{D}}_{i, 1}^{k}+\tilde{\mathcal{I}}_{i, 1}^{k}\right), \\
& \Omega_{i, 1}^{k}=\hbar_{\theta}\left(\frac{\varepsilon_{2}}{\operatorname{Pr}} \hat{I}_{i, 1}^{k-1}+\tilde{\mathcal{L}}_{i, 1}^{k}+\tilde{\mathcal{N}}_{i, 1}^{k}\right)
\end{aligned}
$$

where $\hbar_{f}, \hbar_{g}$, and $\hbar_{\theta}$ are the HAM auxiliary parameters, and

$$
\begin{aligned}
& \hat{D}_{i, j}^{k}=(i+1) \sigma_{i+1, j}^{k} \hat{A}_{i+1, j}^{k}-j \lambda \sigma_{i, j}^{k} \hat{A}_{i, j}^{k} \text {, } \\
& \hat{E}_{i, j}^{k}=(i+1) \sigma_{i+1, j}^{k} \hat{B}_{i+1, j}^{k}-j \lambda \sigma_{i, j}^{k} \hat{B}_{i, j}^{k} \text {, } \\
& \hat{F}_{i, j}^{k}=(i+1) \sigma_{i+1, j}^{k} \hat{C}_{i+1, j}^{k}-j \lambda \sigma_{i, j}^{k} \hat{C}_{i, j}^{k} \text {, } \\
& \hat{G}_{i, j}^{k}=(i+2)(i+1) \sigma_{i+2, j}^{k} \hat{A}_{i+2, j}^{k}-2 j \lambda(i+1) \sigma_{i+1, j}^{k} \hat{A}_{i+1, j}^{k}+(j \lambda)^{2} \sigma_{i, j}^{k} \hat{A}_{i, j}^{k} \text {, } \\
& \hat{H}_{i, j}^{k}=(i+2)(i+1) \sigma_{i+2, j}^{k} \hat{B}_{i+2, j}^{k}-2 j \lambda(i+1) \sigma_{i+1, j}^{k} \hat{B}_{i+1, j}^{k}+(j \lambda)^{2} \sigma_{i, j}^{k} \hat{B}_{i, j}^{k} \text {, } \\
& \hat{I}_{i, j}^{k}=(i+2)(i+1) \sigma_{i+2, j}^{k} \hat{C}_{i+2, j}^{k}-2 j \lambda(i+1) \sigma_{i+1, j}^{k} \hat{C}_{i+1, j}^{k}+(j \lambda)^{2} \sigma_{i, j}^{k} \hat{A}_{i, j}^{k} \text {, } \\
& \hat{J}_{i, j}^{k}=(i+3)(i+2)(i+1) \sigma_{i+3, j}^{k} \hat{A}_{i+3, j}^{k}-3 j \lambda(i+2)(i+1) \sigma_{i+2, j}^{k} \hat{A}_{i+2, j}^{k} \\
& +3(i+1)(j \lambda)^{2} \sigma_{i+1, j}^{k} \hat{A}_{i+1, j}^{k}-(j \lambda)^{3} \sigma_{i, j}^{k} \hat{A}_{i, j}^{k} \text {, } \\
& \hat{K}_{i, j}^{k}=(i+3)(i+2)(i+1) \sigma_{i+3, j}^{k} \hat{B}_{i+3, j}^{k}-3 j \lambda(i+2)(i+1) \sigma_{i+2, j}^{k} \hat{B}_{i+2, j}^{k} \\
& +3(i+1)(j \lambda)^{2} \sigma_{i+1, j}^{k} \hat{B}_{i+1, j}^{k}-(j \lambda)^{3} \sigma_{i, j}^{k} \hat{B}_{i, j}^{k}, \\
& \tilde{\mathcal{A}}_{i, j}^{k}=\sum_{n=0}^{k-1} \sum_{s=\max \{1, j-n-1\}}^{\min \{k-n, j-1\}} \sum_{r=\max \{0, i-n-1\}}^{\max \{k-n, i\}} \sigma_{r, s}^{k-1-n} \hat{A}_{r, s}^{k-1-n} \hat{G}_{i-r, j-s}^{n}, \\
& \tilde{\mathcal{B}}_{i, j}^{k}=\sum_{n=\max \{j-1, i-1\}}^{k-1} \hat{A}_{0,0}^{k-1-n} \hat{G}_{i, j}^{n} \\
& \tilde{\mathcal{C}}_{i, j}^{k}=\sum_{n=0}^{k-1} \sum_{s=\max \{1, j-n-1\}}^{\min \{k-n, j-1\}} \sum_{r=\max \{0, i-n-1\}}^{\max \{k-n, i\}} \sigma_{r, s}^{k-1-n} \hat{A}_{r, s}^{k-1-n} \hat{H}_{i-r, j-s}^{n} \text {, } \\
& \tilde{\mathcal{D}}_{i, j}^{k}=\sum_{n=\max \{j-1, i-1\}}^{k-1} \hat{A}_{0,0}^{k-1-n} \hat{H}_{i, j}^{n}, \\
& \tilde{\mathcal{E}}_{i, j}^{k}=\sum_{n=0}^{k-1} \sum_{s=\max \{1, j-n-1\}}^{\min \{k-n, j-1\}} \sum_{r=\max \{0, i-n-1\}}^{\max \{k-n, i\}} \hat{D}_{r, s}^{k-1-n} \hat{D}_{i-r, j-s}^{n}, \\
& \tilde{\mathcal{F}}_{i, j}^{k}=\sum_{n=0}^{k-1} \sum_{s=\max \{1, j-n-1\}}^{\min \{k-n, j-1\}} \sum_{r=\max \{0, i-n-1\}}^{\max \{k-n, i\}} \sigma_{r, s}^{k-1-n} \hat{B}_{r, s}^{k-1-n} \hat{G}_{i-r, j-s}^{n},
\end{aligned}
$$




$$
\begin{aligned}
\tilde{\mathcal{G}}_{i, j}^{k} & =\sum_{n=\max \{j-1, i-1\}}^{k-1} \hat{B}_{0,0}^{k-1-n} \hat{G}_{i, j}^{n}, \\
\tilde{\mathcal{H}}_{i, j}^{k} & =\sum_{n=0}^{k-1} \sum_{s=\max \{1, j-n-1\}}^{\min \{k-n, j-1\}} \sum_{r=\max \{0, i-n-1\}}^{\max \{k-n, i\}} \sigma_{r, s}^{k-1-n} \hat{B}_{r, s}^{k-1-n} \hat{H}_{i-r, j-s}^{n}, \\
\tilde{\mathcal{I}}_{i, j}^{k} & =\sum_{n=\max \{j-1, i-1\}}^{k-1} \hat{B}_{0,0}^{k-1-n} \hat{H}_{i, j}^{n}, \\
\tilde{\mathcal{J}}_{i, j}^{k} & =\sum_{n=0}^{k-1} \sum_{s=\max \{1, j-n-1\}}^{\min \{k-n, j-1\}} \sum_{r=\max \{0, i-n-1\}}^{\min \{k-n, i\}} \hat{E}_{r, s}^{k-1-n} \hat{E}_{i-r, j-s}^{n}, \\
\tilde{\mathcal{K}}_{i, j}^{k} & =\sum_{n=0}^{k-1} \sum_{s=\max \{1, j-n-1\}}^{\min \{k-n, j-1\}} \sum_{\max \{k-n, i\}} \sigma_{r, s}^{k-1-n} \hat{A}_{r, s}^{k-1-n} \hat{F}_{i-r, j-s}^{n}, \\
\tilde{\mathcal{L}}_{i, j}^{k} & =\sum_{n=\max \{j-1, i-1\}}^{k-1} \hat{A}_{0,0}^{k-1-n} \hat{F}_{i, j}^{n}, \\
\tilde{\mathcal{M}}_{i, j}^{k} & =\sum_{n=0}^{k-1} \sum_{\min \{k-n, j-1\}} \sum_{\max \{k-n, i\}} \sum_{\tilde{\mathcal{N}}_{i, j}^{k}}^{k}=\sum_{n=\max \{1, j-n-1\}}^{k-1} \sigma_{r=\max \{0, i-n-1\}}^{k-1, i-1\}} \hat{B}_{0,0}^{k-1-n} \hat{F}_{i, j}^{n} .
\end{aligned}
$$

When we set $A_{0,0}^{0}=1 / \lambda, A_{0,1}^{0}=-1 / \lambda, B_{0,0}^{0}=c / \lambda, B_{0,1}^{0}=-c / \lambda$, and $C_{0,1}^{0}=1$, all coefficients in the above formulas can be determined successively in the order $k=1,2,3, \ldots$.

\section{Results}

As mentioned before, the explicit solutions for $f(\eta), g(\eta)$, and $\theta(\eta)$ have been obtained. In this part, we shall first provide tools for the choice of the HAM auxiliary parameter $\hbar$. Then we shall give the method for error verification of the obtained results. The behavior at far field of these solutions will be checked subsequently. At the end, the effect of the solid volume fraction of the nanofluid $\phi$ on the velocity and temperature, as well as the wall friction and the Nusselt number shall be presented and discussed.

\subsection{Techniques for choice of $\hbar$ and error verification}

We now present the technique for choice of $\hbar$. For the purpose of selection of the optimal values of the auxiliary parameters $\hbar_{f}, \hbar_{g}$, and $\hbar_{\theta}$, we define the following three error functions:

$$
\begin{aligned}
& X \text { Err }=\lim _{l \rightarrow \infty} \int_{0}^{l}\left[\varepsilon_{1} f^{\prime \prime \prime}+(f+g) f^{\prime \prime}-\left(f^{\prime}\right)^{2}\right]^{2} d \eta, \\
& Y \text { Err }=\lim _{l \rightarrow \infty} \int_{0}^{l}\left[\varepsilon_{1} g^{\prime \prime \prime}+(f+g) g^{\prime \prime}-\left(g^{\prime}\right)^{2}\right]^{2} d \eta, \\
& T \text { Err }=\lim _{l \rightarrow \infty} \int_{0}^{l}\left[\frac{\varepsilon_{2}}{\operatorname{Pr}} \theta^{\prime \prime}+(f+g) \theta^{\prime}\right]^{2} d \eta,
\end{aligned}
$$

where $l$ is the integration distance and it is found that $l=100$ is adequate in our calculation. 


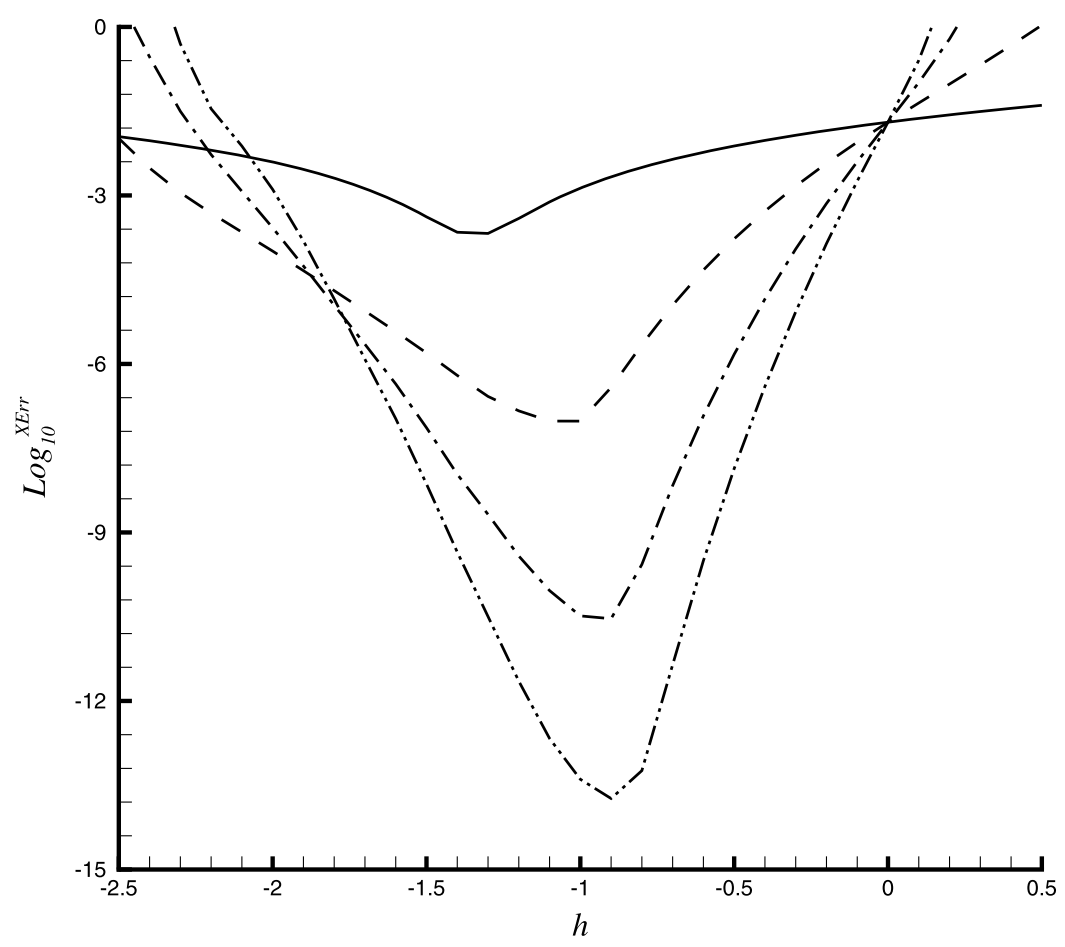

Figure $2 \hbar$ curve for (73). Solid line: first-order approximation. Dashed line: fifth-order approximation. Dash-dotted line: tenth-order approximation. Dash-dot-dotted line: 15th-order approximation.

For the prescribed values of physical parameters $\varepsilon_{1}, \varepsilon_{2}$, and $\operatorname{Pr}$, (73)-(75) are functions of $\hbar_{f}, \hbar_{g}$, and $\hbar_{\theta}$ as a certain order HAM approximations are substituted. The optimal values of $\hbar_{f}, \hbar_{g}$, and $\hbar_{\theta}$ can then be obtained by evaluating the least errors of (73)-(75). In order to reduce the computation quantities, we set $\hbar_{f}=\hbar_{g}=\hbar_{\theta}=\hbar$. In this situation, the optimal value of $\hbar_{f}$ corresponds to the least error of (73), the optimal value of $\hbar_{g}$ corresponds to the least error of (74), and the optimal value of $\hbar_{\theta}$ corresponds to the least error of (75), respectively. By means of this approach [34], we are able to determine the optimal values of $\hbar_{f}, \hbar_{g}$, and $\hbar_{\theta}$ by plotting the $\hbar$ curves, as shown in Figures $2-4$. Here $\operatorname{Pr}=1$ is chosen. From these figures, it is found that the appropriate HAM auxiliary parameter(s) $\hbar$ can always help to reduce the residual errors of the HAM analytical approximations. This can be deemed as the principal rule for the evaluation of the validity and correctness of the selected value(s) of $\hbar$.

We then check the accuracies of these explicit solutions. Firstly, we use BVPh 2.0 to solve this problem and compare the results with HAM approximations (see Figure 5). It shows that the results of HAM are valid. Particularly for $\phi=0$, this problem is identical to Wang's problem [35]. In this situation, we compare our analytical approximations for $f^{\prime \prime}(0), g^{\prime \prime}(0), f(\infty)$, and $g(\infty)$ with Wang's results [35], and very excellent agreement is found, as shown in Table 2. On the other hand, it is convenient to obtain the residual errors of these HAM approximations by means of (73)-(75) as well. As seen from Table 3, for carefully selected optimal values of $\hbar_{f}=\hbar_{g}=\hbar_{\theta}=-1$, the logarithms of the residual errors decrease dramatically with increasing of the HAM computational orders. This provides us with numerical evidence that our series solutions are convergent. In our computation, all the residual errors are less than $1.0 \times 10^{-7}$. 


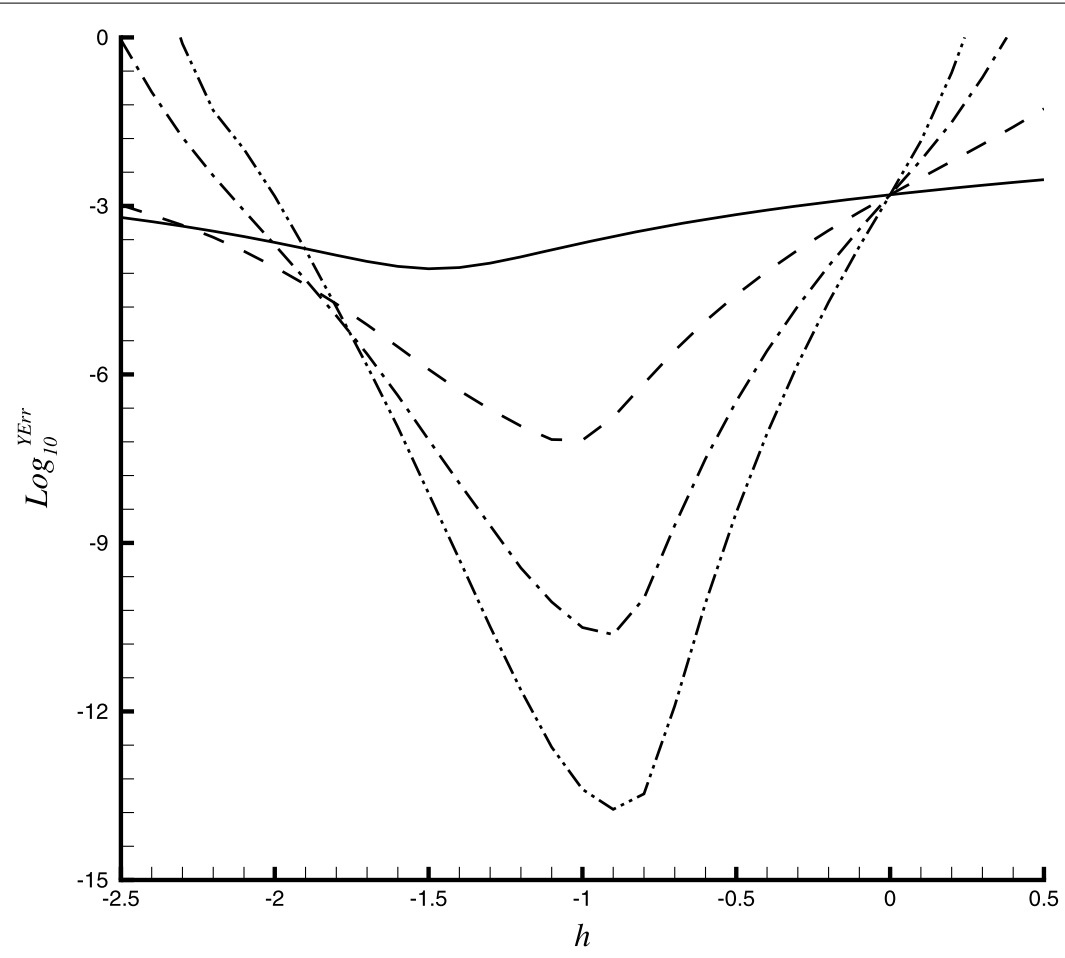

Figure $3 \hbar$ curve for (74). Solid line: first-order approximation. Dashed line: fifth-order approximation. Dash-dotted line: tenth-order approximation. Dash-dot-dotted line: 15th-order approximation.

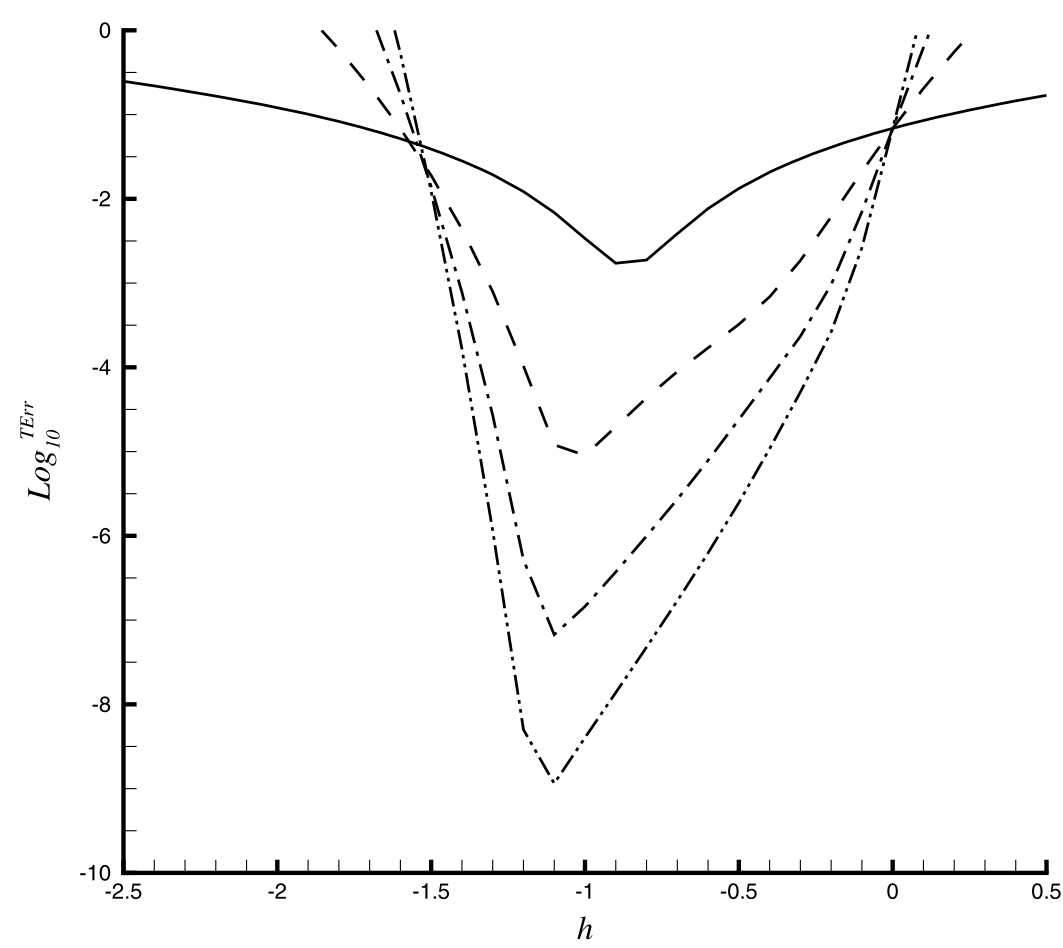

Figure 4 i curve for (75). Solid line: first-order approximation. Dashed line: fifth-order approximation. Dash-dotted line: tenth-order approximation. Dash-dot-dotted line: 15th-order approximation. 


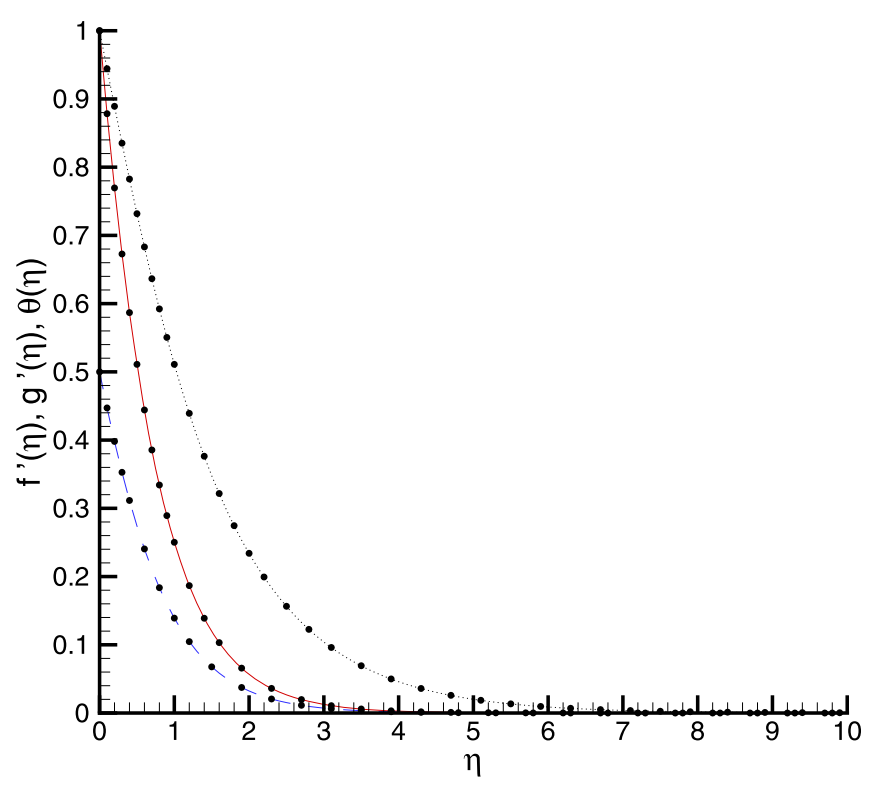

Figure 5 Comparison of the BVPh 2.0 results with the HAM results in case of $c=1 / 2, \lambda=1$ and $\phi=0.1$. Symbols: BVPh 2.0 results. The HAM results: solid line: $f^{\prime}(\eta)$, dashed line: $g^{\prime}(\eta)$, dotted line: $\theta(\eta)$.

Table 2 Comparison of the 20th-order HAM approximations with Wang's results [35] (marked with an asterisk)

\begin{tabular}{lrllll}
\hline $\boldsymbol{c}$ & $\mathbf{0}$ & $\mathbf{0 . 2 5}$ & $\mathbf{0 . 5 0}$ & $\mathbf{0 . 7 5}$ & $\mathbf{1}$ \\
\hline$f^{\prime \prime}(0)$ & -1 & -1.048811 & -1.093095 & -1.134486 & -1.173721 \\
$f^{\prime \prime}(0)^{*}$ & -1 & -1.048813 & -1.093097 & -1.134485 & -1.173720 \\
$g^{\prime \prime}(0)$ & 0 & -0.194564 & -0.465205 & -0.794618 & -1.173721 \\
$g^{\prime \prime}(0)^{*}$ & 0 & -0.194564 & -0.465205 & -0.794622 & -1.173720 \\
$f(\infty)$ & 1 & 0.907151 & 0.842386 & 0.792302 & 0.751497 \\
$f(\infty)^{*}$ & 1 & 0.907075 & 0.842360 & 0.792308 & 0.751527 \\
$g(\infty)$ & 0 & 0.257994 & 0.451678 & 0.612135 & 0.751497 \\
$g(\infty)^{*}$ & 0 & 0.257986 & 0.451671 & 0.612049 & 0.751527 \\
\hline
\end{tabular}

Table 3 Residual errors for various order HAM computation with $\hbar=-1, c=0.5$ and $\operatorname{Pr}=1$

\begin{tabular}{clll}
\hline $\boldsymbol{k}$ th-order & $\boldsymbol{X}$ Err & $\boldsymbol{Y}$ Err & $\boldsymbol{T}$ Err \\
\hline 1 & $1.3778 \times 10^{-3}$ & $2.2158 \times 10^{-4}$ & $4.2787 \times 10^{-3}$ \\
5 & $9.6968 \times 10^{-8}$ & $6.7691 \times 10^{-8}$ & $9.1674 \times 10^{-6}$ \\
10 & $3.2657 \times 10^{-11}$ & $3.1218 \times 10^{-11}$ & $1.4527 \times 10^{-7}$ \\
15 & $4.0317 \times 10^{-14}$ & $4.1084 \times 10^{-14}$ & $4.0237 \times 10^{-9}$ \\
20 & $7.0457 \times 10^{-17}$ & $7.3800 \times 10^{-17}$ & $1.5154 \times 10^{-10}$ \\
\hline
\end{tabular}

The nanofluid is Cu-water.

\subsection{Asymptotic analysis}

We now examine the asymptotic behaviors of the velocity profiles $f^{\prime}(\eta)$ and $g^{\prime}(\eta)$, and the temperature profile $s(\eta)$ at far field. According to the boundary conditions (12) and (13), for considerably large $\eta$, it is well known that

$$
\lim _{\eta \rightarrow \infty} f^{\prime} \rightarrow 0, \quad \lim _{\eta \rightarrow \infty} g^{\prime} \rightarrow 0, \quad \lim _{\eta \rightarrow \infty} \theta \rightarrow 0
$$


Integrating $f^{\prime}$ and $g^{\prime}$ with $\eta$ once, we obtain

$$
\lim _{\eta \rightarrow \infty} f \rightarrow \alpha_{1}, \quad \lim _{\eta \rightarrow \infty} g \rightarrow \alpha_{2},
$$

where $\alpha_{1}$ and $\alpha_{2}$ are integral constants. For physical requirements $f^{\prime} \geq 0$ and $g^{\prime} \geq 0$, it is readily seen that $\alpha_{1} \geq 0$ and $\alpha_{2} \geq 0$.

For considerably large $\eta$, we suppose $f, g$, and $s$ can be expressed by

$$
f=\alpha_{1}+F, \quad g=\alpha_{2}+G, \quad \theta=S,
$$

where $F, G$, and $S$ are negligibly small.

Substituting (77) into (9)-(11) and linearizing them, we obtain

$$
\begin{aligned}
& \varepsilon_{1} F^{\prime \prime \prime}+\alpha_{3} F^{\prime \prime}=0, \\
& \varepsilon_{1} G^{\prime \prime \prime}+\alpha_{3} G^{\prime \prime}=0, \\
& \frac{\varepsilon_{2}}{P r} S^{\prime \prime}+\alpha_{3} S^{\prime}=0,
\end{aligned}
$$

where

$$
\alpha_{3}=\alpha_{1}+\alpha_{2} \geq 0
$$

Due to (76) and (77),

$$
F^{\prime}=G^{\prime}=S=0 \quad \text { as } \eta \rightarrow \infty .
$$

Equations (78)-(80) are linear equations with constant coefficients, their solutions can be explicitly given as

$$
F^{\prime}=G^{\prime}=C_{1} \exp \left(-\frac{\alpha_{3}}{\varepsilon_{1}} \eta\right)+C_{2}
$$

where $C_{1}$ and $C_{2}$ are integral constants. Based on the boundary condition (82) for $F^{\prime}$ and $G^{\prime}$ at far field, it is readily seen that $C_{2}=0$. Equation (83) is then reduced to

$$
F^{\prime}=G^{\prime}=C_{1} \exp \left(-\frac{\alpha_{3}}{\varepsilon_{1}} \eta\right)
$$

Similarly, due to (80) and its boundary condition (82), we obtain

$$
S=C_{3} \exp \left(-\frac{\alpha_{3} P r}{\varepsilon_{2}} \eta\right)
$$

where $C_{3}$ is an integral constant. It can be seen from (84) and (85) that $F^{\prime}, G^{\prime}$, and $S$ (hence $f^{\prime}, g^{\prime}$, and $s$ ) approach zero in an exponential decaying manner as $\eta \rightarrow \infty$.

\subsection{Effects of the nanoparticle volume fraction $\phi$ on the flows}

It is well known that $\phi$ is the physical quantity for description of the volumetric ratio of solid nanoparticles in nanofluids. $\phi=0$ denotes the pure fluid (Newtonian fluid) and $\phi \geq 0$ 


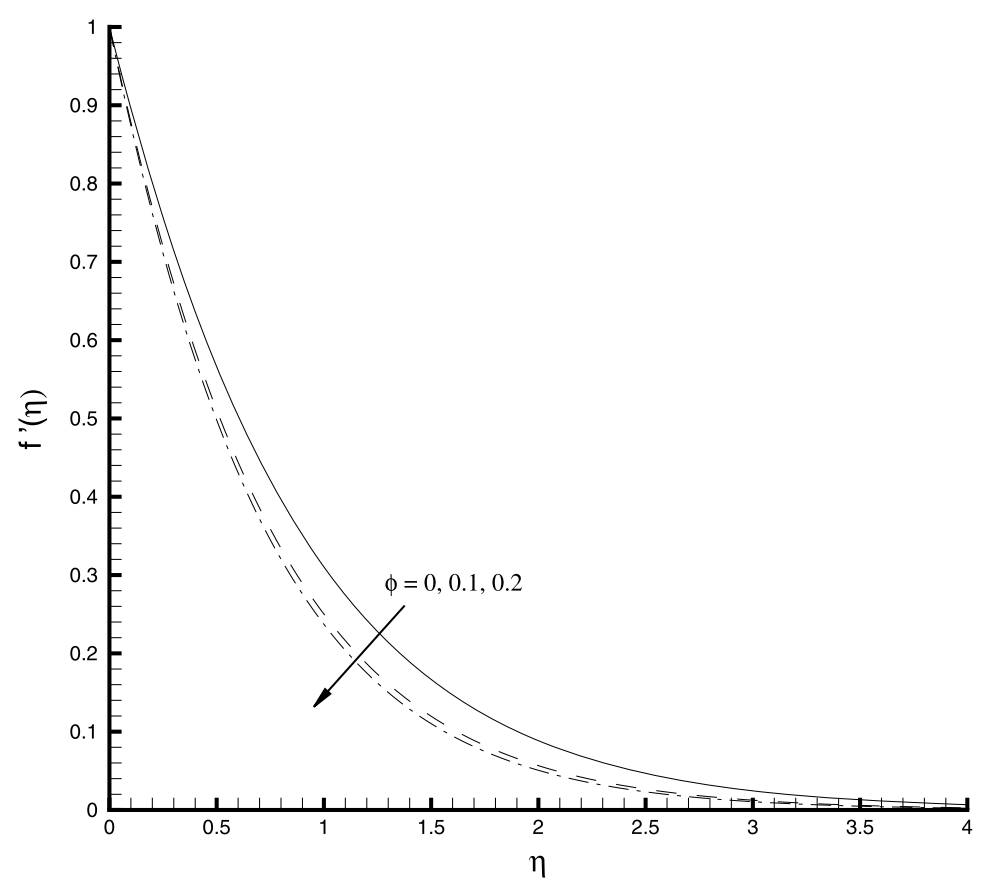

Figure 6 The velocity profiles $f^{\prime}(\eta)$ for various values of $\phi$ with $c=1 / 2$. Solid line: $\phi=0$; dashed line: $\phi=0.1$; dash-dotted line: $\phi=0.2$.

denotes the nanofluids. We now investigate its influence on the velocity and temperature distributions, as well as the reduced local skin friction coefficients and the reduced Nusselt numbers of the considered boundary-layer flows.

It is found in Figures 6 and 7 that the velocity profiles $f^{\prime}(\eta)$ and $g^{\prime}(\eta)$ decrease continuously as $\eta$ increases for all considered $\phi$. It is further found that $f^{\prime}(\eta)$ and $g^{\prime}(\eta)$ diminish more quickly for larger values of $\phi$. The descending trend becomes slower and slower as $\phi$ enlarges. This is due to the boundary layer thickness being proportional to the square root of kinematic viscosity, which is expressed by $v_{\mathrm{nf}}=\mu_{\mathrm{nf}} / \rho_{\mathrm{nf}}$ in nanofluids. According to the definitions of (7), we have $v_{\mathrm{nf}} \approx 0.725 v_{\mathrm{f}}$ for $\phi=0.1$ and $v_{\mathrm{nf}} \approx 0.674 v_{\mathrm{f}}$ for $\phi=0.2$. Therefore, the thickness of the velocity boundary layer of the nanofluid is less than that of the pure fluid.

The variation of the temperature profiles $\theta(\eta)$ with $\eta$ for various values of $\phi$ is plotted in Figure 8 . Similar to the trend of the velocity variation, $\theta(\eta)$ decreases monotonously with the increase of $\eta$. While $\phi$ exhibits a completely different effect on the temperature variation, the larger the value of $\phi$, the higher is the temperature profile. This can be explained by the nanofluids having the superior thermal conductivity since the metallic particles, one of the components of the nanofluid, have better thermal conductivity than pure fluids. As a result, the heat transfer capability of the fluids is improved significantly, which leads to the enhancement of the temperature profile.

We then consider the influences of the nanoparticle volume fraction $\phi$ on the local skin friction coefficients and the local Nusselt numbers. It is shown by Figure 9 that both the reduced local skin friction coefficients $R e_{x}^{1 / 2} C_{f x}$ and $R e_{y}^{1 / 2} C_{f y}$ decrease monotonously with $\phi$ increasing from 0 to 0.3 . This indicates that an increase of the concentration of nanoparticles results in an enlargement of the skin friction in the opposite direction of the wall 


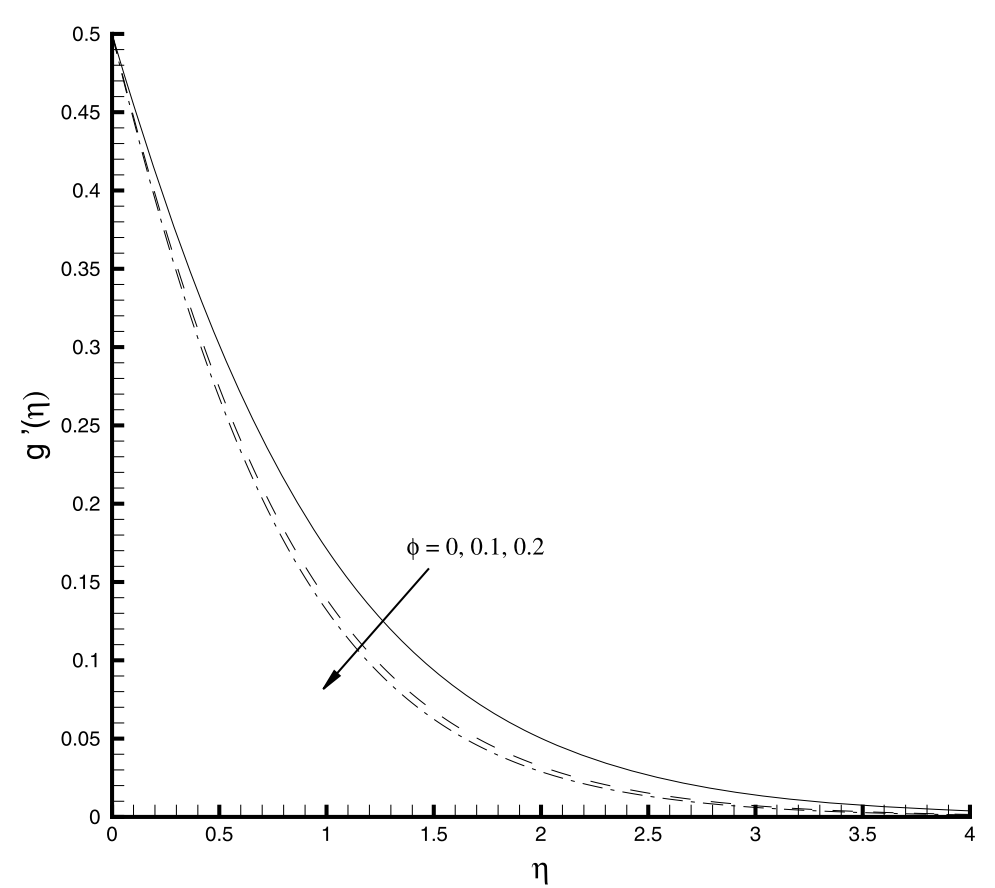

Figure 7 The velocity profiles $g^{\prime}(\eta)$ for various values of $\phi$ with $c=1 / 2$. Solid line: $\phi=0$; dashed line: $\phi=0.1$; dash-dotted line: $\phi=0.2$.

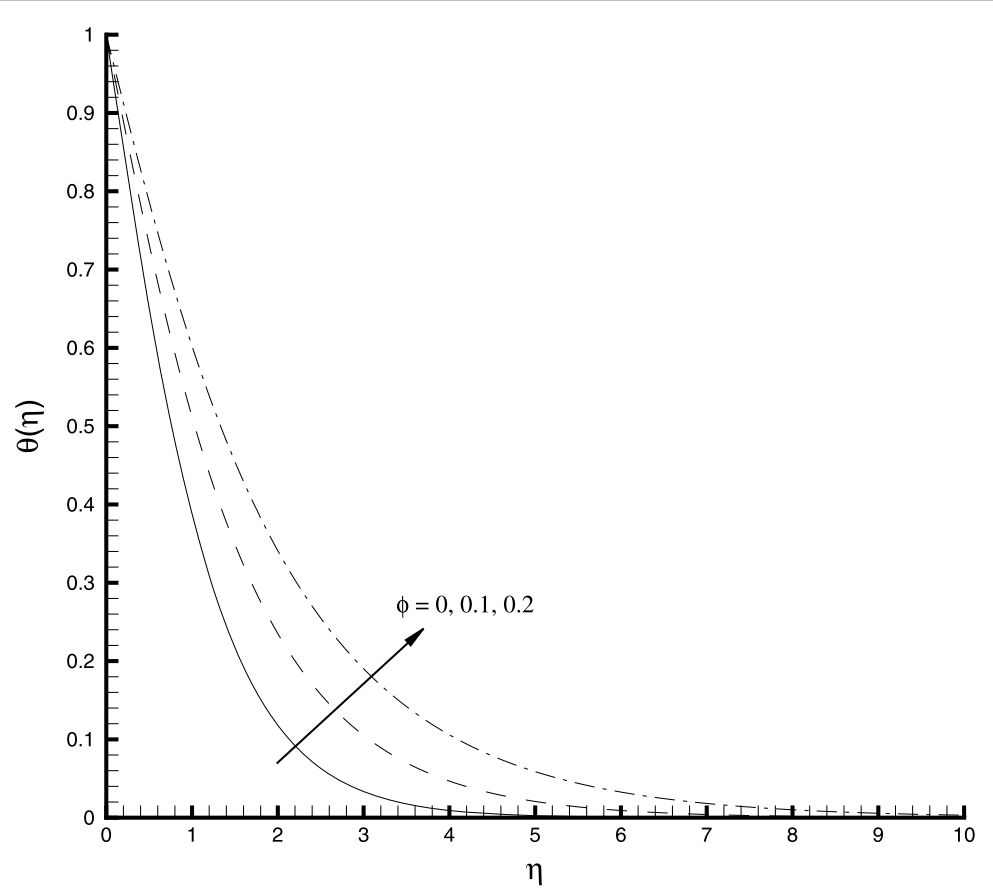

Figure 8 The temperature profiles $\theta(\eta)$ for various values of $\phi$ with $c=1 / 2$. Solid line: $\phi=0$; dashed line: $\phi=0.1$; dash-dotted line: $\phi=0.2$. 


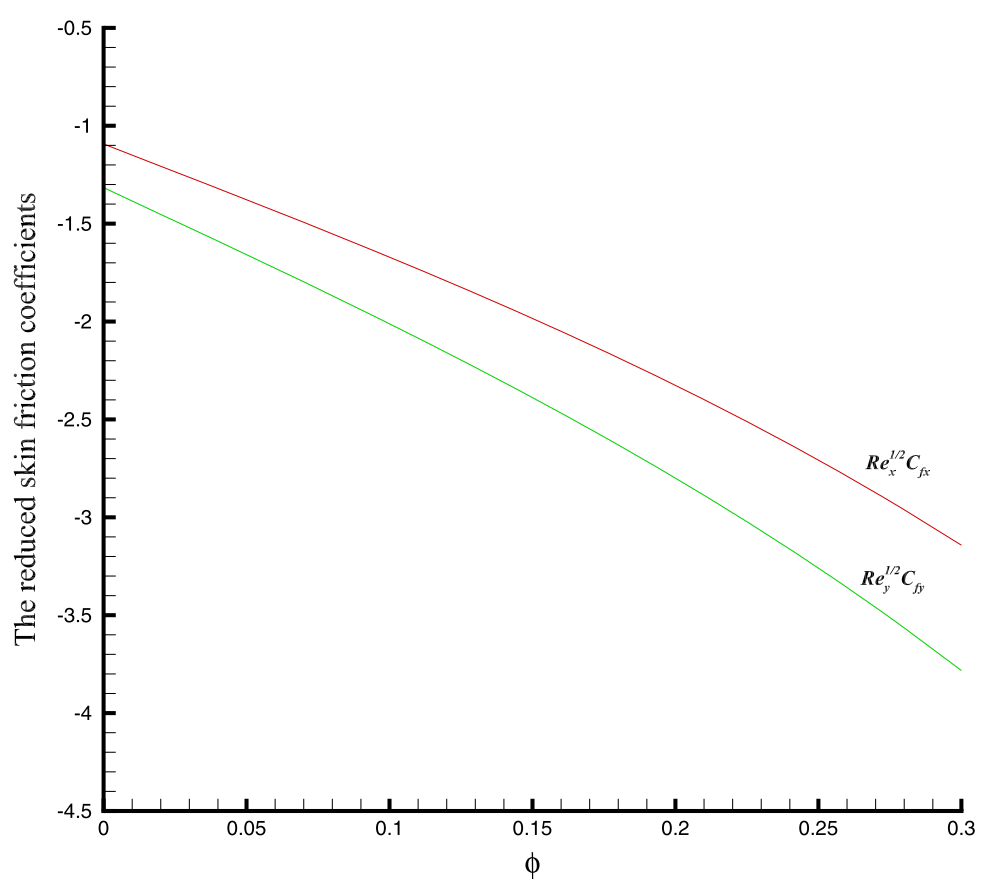

Figure 9 Variation of the reduced local skin friction coefficients with $\phi$ for $c=1 / 2$.

stretching. Based on (16), we further find that the local skin friction coefficient in the $x$ direction is independent of the parameter $c$, while the local skin friction coefficient in the $y$-direction relies on $c$ and its value is inversely proportional to $c^{3 / 2}$. This means the larger the value of $c$, the smaller is the local skin friction coefficient $R e_{y}^{1 / 2} C_{f y}$.

Similar to the changing trend of the local skin friction coefficients, the local Nusselt numbers $R e_{x}^{1 / 2} N u_{x}$ and $R e_{y}^{1 / 2} N u_{y}$ decrease continuously as $\phi$ evolves, but their descendent rates are slower than those of the local skin friction coefficients, as shown in Figure 10. This can be deemed to be due to the increase of the nanoparticle volume fraction $\phi$ helping to increase the ratio of convective to conductive heat transfer across the boundary. This phenomenon again is evidence that the heat transfer conductivity of nanofluids is superior to that of pure fluids. Also from (16), we find that the local Nusselt number in the $x$-direction has no relation with the parameter $c$, but the local skin friction coefficient in the $y$-direction is dependent on $c$ and its value is inversely proportional to $c^{1 / 2}$.

\section{Conclusions}

In this paper, the three-dimensional nanofluid flow and heat transfer in the boundary-layer region over a flat sheet stretched continuously in two lateral directions has been examined in detail. The explicit solutions for this flow problem have been obtained by means of the homotopy analysis method, which exhibits the exponentially decaying behaviors at far field. Besides, the effects of the nanoparticle volume fraction $\phi$ on the velocity and temperature profiles, as well as the reduced local skin friction coefficients and the reduced local Nusselt numbers have been investigated. It is found that the heat transfer conductivity of the base fluid can be enhanced significantly as the appropriate metallic particles in nano-scale are added into the pure fluids. We believe that this work paves the way for a systematic experimental investigation of the three-dimensional nanofluids flow and heat 


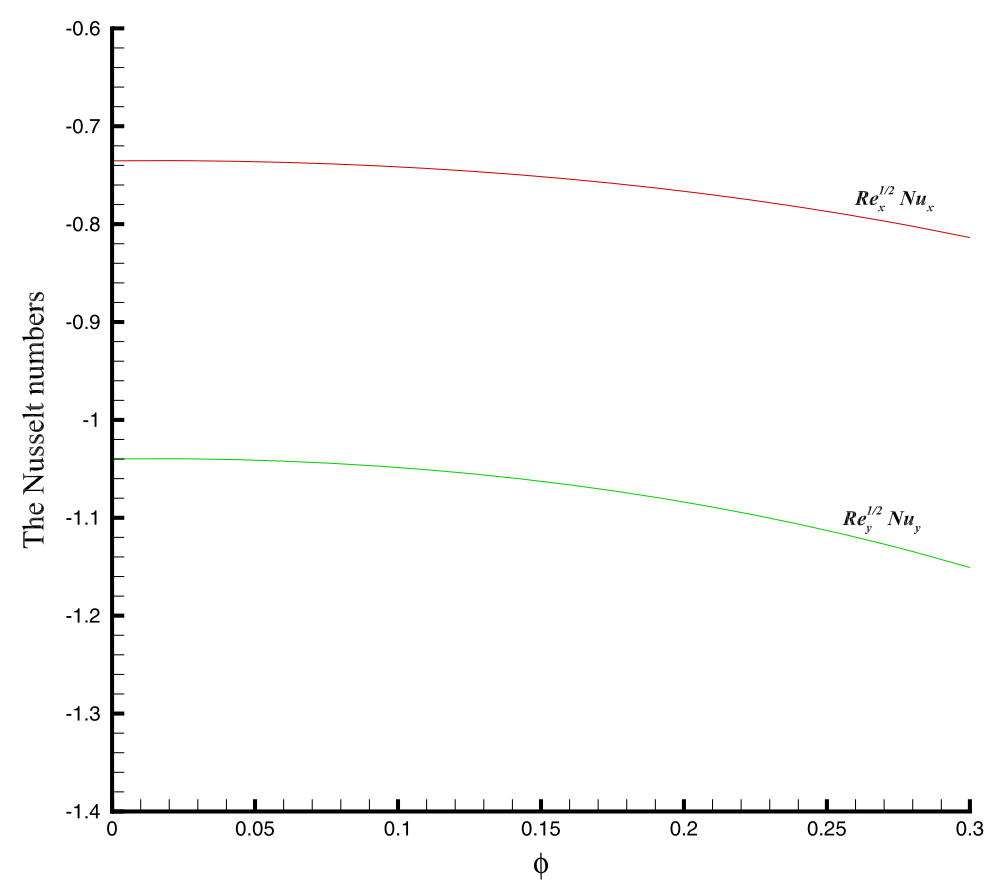

Figure 10 Variation of the reduced local Nusselt numbers with $\phi$ for $c=1 / 2$ and $\operatorname{Pr}=1$.

transfer over a stretching surface. We also believe this work can help researchers to derive explicit solutions of other boundary layer problems.

\section{Competing interests}

The authors declare that they have no competing interests.

\section{Authors' contributions}

All authors contributed equally to the writing of this paper. All authors read and approved the final manuscript.

\section{Author details}

'State Key Lab of Ocean Engineering, School of Naval Architecture, Ocean and Civil Engineering, Shanghai Jiao Tong University, Shanghai, 200240, P.R. China. ${ }^{2}$ Present address: Post-Doctoral Research Station, Jiangnan Shipyard Group Co., Ltd, Shanghai, 201913, P.R. China.

\section{Acknowledgements}

We express our sincere appreciations for reviewers' valuable comments and suggestions. We extend our sincere appreciations to the Program for New Century Excellent Talents in University (Grant No. NCET-12-0347) for its financial support.

Received: 7 January 2015 Accepted: 8 April 2015 Published online: 22 April 2015

\section{References}

1. Crane, L: Flow past a stretching plate. Z. Angew. Math. Phys. 21, 645-647 (1970)

2. Gupta, PS, Gupta, AS: Heat and mass transfer on a stretching sheet with suction or blowing. Can. J. Chem. Eng. 55(6), 744-746 (1977)

3. Chakrabarti, A, Gupta, AS: Hydromagnetic flow and heat transfer over a stretching sheet. Q. Appl. Math. 37(1), 73-78 (1979)

4. Carragher, P, Crane, LJ: Heat transfer on a continuous stretching sheet. Z. Angew. Math. Mech. 62(10), 564-565 (1982)

5. Dutta, BK, Roy, P, Gupta, AS: Temperature field in flow over a stretching sheet with uniform heat flux. Int. Commun. Heat Mass Transf. 12(1), 89-94 (1985)

6. Jeng, DR, Chang, TCA, De Witt, KJ: Momentum and heat transfer on a continuous moving surface. J. Heat Transf. 108(3), 532-539 (1986)

7. Dutta, BK: Heat transfer from a stretching sheet with uniform suction and blowing. Acta Mech. 78(3-4), 255-262 (1989)

8. Andersson, $\mathrm{HI}$ : An exact solution of the Navier-Stokes equations for magnetohydrodynamic flow. Acta Mech. 113(1-4), 241-244 (1995) 
9. Chiam, TC: Heat transfer with variable conductivity in a stagnation-point flow towards a stretching sheet. Int. Commun. Heat Mass Transf. 23(2), 239-248 (1996)

10. Vajravelu, K, Hadjinicolaou, A: Convective heat transfer in an electrically conducting fluid at a stretching surface with uniform free stream. Int. J. Eng. Sci. 35(12-13), 1237-1244 (1997)

11. Wang, CY: The three-dimensional flow due to a stretching flat surface. Phys. Fluids 27(8), 1915-1917 (1984)

12. Hayat, T, Sajid, M, Pop, I: Three-dimensional flow over a stretching surface in a viscoelastic fluid. Nonlinear Anal., Real World Appl. 9(4), 1811-1822 (2008)

13. Takhar, HS, Chamkha, AJ, Nath, G: Unsteady three-dimensional MHD-boundary-layer flow due to the impulsive motion of a stretching surface. Acta Mech. 146, 59-71 (2001)

14. Choi, SUS: Enhancing thermal conductivity of fluids with nanoparticle. In: The Proceedings of the 1995 ASME International Mechanical Engineering Congress and Exposition, San Francisco, USA, ASME, FED 231/MD 66, pp. 99-105 (1995)

15. Maïga, SEB, Palm, SJ, Nguyen, CT, Roy, G, Galanis, N: Heat transfer enhancement by using nanofluids in forced convection flows. Int. J. Heat Fluid Flow 26(4), 530-546 (2005)

16. Xuan, YM, Roetzel, W: Conceptions for heat transfer correlation of nanofluids. Int. J. Heat Mass Transf. 43(19) 3701-3707 (2000)

17. Buongiorno, J: Convective transport in nanofluids. J. Heat Transf. 128(3), 240-250 (2006)

18. Nield, DA, Kuznetsov, AV: The Cheng-Minkowycz problem for natural convective boundary-layer flow in a porous medium saturated by a nanofluid. Int. J. Heat Mass Transf. 52, 5792-5795 (2009)

19. Nield, DA, Kuznetsov, AV: The Cheng-Minkowycz problem for the double-diffusive natural convective boundary layer flow in a porous medium saturated by a nanofluid. Int. J. Heat Mass Transf. 54, 374-378 (2011)

20. Cheng, P, Minkowycz, WJ: Free convection about a vertical flat plate embedded in a porous medium with application to heat transfer from a dike. J. Geophys. Res. 82, 2040-2044 (1977)

21. Yacob, NA, Ishak, A, Pop, I, Vajravelu, K: Boundary layer flow past a stretching/shrinking surface beneath an external uniform shear flow with a convective surface boundary condition in a nanofluid. Nanoscale Res. Lett. 6, 314-320 (2011)

22. Bachok, N, Ishak, A, Pop, I: Flow and heat transfer over a rotating porous disk in a nanofluid. Physica B 406, 1767-1772 (2011)

23. Rohni, AM, Ahmad, S, Pop, l: Flow and heat transfer over an unsteady shrinking sheet with suction in nanofluids. Int. J. Heat Mass Transf. 55, 1888-1895 (2012)

24. Yacob, NA, Ishak, A, Pop, I: Falkner-Skan problem for a static or moving wedge in nanofluids. Int. J. Therm. Sci. 50, 133-139 (2011)

25. Vajravelu, K, Prasad, KV, Lee, J, Lee, C, Pop, I, Vab Gorder, RA: Convective heat transfer in the flow of viscous Ag-water and Cu-water nanofluids over a stretching surface. Int. J. Therm. Sci. 50, 843-851 (2011)

26. Nield, DA, Kuznetsov, AV: The onset of convection in a horizontal nanofluid layer of finite depth. Eur. J. Mech. B, Fluids 29, 217-223 (2010)

27. Kuznetsov, AV, Nield, DA: Natural convective boundary-layer flow of a nanofluid past a vertical plate. Int. J. Therm. Sci. 49, 243-247 (2010)

28. Kuznetsov, AV, Nield, DA: Double-diffusive natural convective boundary-layer flow of a nanofluid past a vertical plate Int. J. Therm. Sci. 50, 712-717 (2011)

29. Khan, WA, Pop, I: Boundary-layer flow of a nanofluid past a stretching sheet. Int. J. Heat Mass Transf. 53, 2477-2483 (2010)

30. Liao, SJ: Beyond Perturbation: Introduction to the Homotopy Analysis Method. Chapman \& Hall/CRC, Boca Raton (2003)

31. Xu, H, Fan, T, You, XC: Three-dimensional boundary-layer flow and heat transfer of a Cu-water nanofluid over a stretching surface. In: International Conference of Numerical Analysis and Applied Mathematics, Kos, Greece. AIP Conference Proceedings, vol. 1479, pp. 1845-1849 (2012)

32. Brinkman, HC: The viscosity of concentrated suspensions and solutions. J. Chem. Phys. 20, 571-581 (1952)

33. Oztop, HF, Abu-Nada, E: Numerical study of natural convection in partially heated rectangular enclosures filled with nanofluids. Int. J. Heat Fluid Flow 29, 1326-1336 (2008)

34. Liao, SJ: Homotopy Analysis Method in Nonlinear Differential Equations. Higher Education Press, Beijing (2012)

35. Wang, CY: The three-dimensional flow due to a stretching flat surface. Phys. Fluids 27, 1915-1917 (1984)

\section{Submit your manuscript to a SpringerOpen ${ }^{\mathcal{O}}$ journal and benefit from:}

- Convenient online submission

Rigorous peer review

- Immediate publication on acceptance

Open access: articles freely available online

- High visibility within the field

- Retaining the copyright to your article 